Research Paper

\title{
Post-natal development of the envelope following response to amplitude modulated sounds in the bat Phyllostomus discolor
}

\author{
Stephen Gareth Hörpel *, Uwe Firzlaff \\ Department of Animal Sciences, Technical University of Munich, Liesel-Beckmann-Str. 4, 85354, Freising, Germany
}

\section{A R T I C L E I N F O}

\section{Article history:}

Received 4 November 2019

Received in revised form

20 December 2019

Accepted 24 January 2020

Available online 30 January 2020

\section{Keywords:}

Temporal processing

Auditory brainstem responses

Amplitude modulation

Hearing development

\begin{abstract}
A B S T R A C T
Bats use a large repertoire of calls for social communication, which are often characterized by temporal amplitude and frequency modulations. As bats are considered to be among the few mammalian species capable of vocal learning, the perception of temporal sound modulations should be crucial for juvenile bats to develop social communication abilities. However, the post-natal development of auditory processing of temporal modulations has not been investigated in bats, so far. Here we use the minimally invasive technique of recording auditory brainstem responses to measure the envelope following response (EFR) to sinusoidally amplitude modulated noise (range of modulation frequencies: $11-130 \mathrm{~Hz}$ ) in three juveniles (p8-p72) of the bat, Phyllostomus discolor. In two out of three animals, we show that although amplitude modulation processing is basically developed at p8, EFRs maturated further over a period of about two weeks until p33. Maturation of the EFR generally took longer for higher modulation frequencies $(87-130 \mathrm{~Hz})$ than for lower modulation frequencies $(11-58 \mathrm{~Hz})$.
\end{abstract}

() 2020 Elsevier B.V. All rights reserved.

\section{Introduction}

Human speech and animal communication sounds are characterized by, among other sound features, temporal amplitude and frequency modulations (Gaucher et al., 2013; Nourski and Brugge, 2011). This holds also true for species-specific communication in bats, which exhibit a rich vocal repertoire (Bohn et al., 2008; Knörnschild, 2014; Knörnschild et al., 2014; Lattenkamp et al., 2019). Furthermore, bats are among the few mammals species considered to be capable of vocal learning (Boughman, 1998; Knörnschild, 2014; Prat et al., 2015). For the bat Phyllostomus discolor, it has been reported that contact calls important for mother infant communication show a less prominent amount of temporal modulations in hand-reared pups compared to pups reared together with their mothers (Esser and Schmidt, 1989). Therefore, the ontogeny of the auditory system in these bats should reflect the need for processing of temporal sound modulations.

Generally, hearing onset is defined as the point during the maturation of an animal, where behavioral (Moore, 1982) or neuronal (Sonntag et al., 2009) responses can by elicited by sound pressure levels within physiological ranges. Common model

\footnotetext{
* Corresponding author.

E-mail address: stephen.hoerpel@tum.de (S.G. Hörpel).
}

animals are typically born deaf, e.g. gerbils (hearing onset at p11p12, Heil et al., 1995; Magnusson et al., 2005), rats (hearing onset at p11, Smith et al., 2000) and mice (hearing onset at p12, Mikaelian and Ruben, 1965; Kraus and Aulbach-Kraus, 1981). In bats, hearing onset seems to vary between different species. However, for most species hearing onset is reported to occur within the first week if not as early as on postnatal day p1-p2 (p7-p8, Antrozous pallidus, Brown et al., 1978; Razak and Fuzessery, 2007; p1-p2, Pteronotus parnellii, Vater et al., 2010 and Carollia perspicillata, Sterbing, 2002; first and second postnatal week, Hipposideros speoris \& Rhinolophus rouxi, Rübsamen et al., 1989). P. discolor was shown to react to maternal directive calls within the first day after birth (Esser and Schmidt, 1990), which is supported by data from Linnenschmidt and Wiegrebe (2019), who showed that hearing onset in this bat has already occurred at p5. It should certainly be clear, that an early hearing onset is of advantage in mother-pup communication.

The post-natal development of processing of temporal modulations has been already investigated in several mammalian species, e.g. gerbils (Heil et al., 1995; Khurana et al., 2012), mice (Müller et al., 2019), harbour porpoises (Linnenschmidt et al., 2013) and in humans (Draganova et al., 2018; Rance et al., 2006; Walker et al., 2019). However, data for bats are lacking so far. In order to analyze the development of temporal modulation processing ability in bats, we recorded envelope following responses (EFRs) from pups of $P$. discolor. 
The EFR is a subclass of auditory brainstem response (ABR) evoked by periodic auditory stimuli. As the EFR is typically phaselocked to the waveform and/or the envelope of a periodic stimulus (Moushegian et al., 1973), it has been used in animals as well as in humans to quantify the temporal processing abilities of the auditory system (Dimitrijevic et al., 2016; Prado-Gutierrez et al., 2012; Venkataraman and Bartlett, 2013).

More generally, the ABR signal reflects the summed electrical response potentials of both the auditory nerve fibers and nuclei of the ascending auditory pathway (Burkard et al., 2007). ABR recordings are ideal for measuring ontogenetic changes of the auditory pathway for mainly two reasons: first, the ABR waveform generally changes substantially during the ontogeny of the animal, reflecting the changes during the maturation of the auditory pathway. Second, being a minimally invasive recording method, it allows for long-term observations from the same animal and is especially suited for new porn pups, which might not be suitable for more invasive techniques.

ABR recordings have been utilized in bat auditory research several times (e.g. Myotis lucifugus \& Plecotus townsendii, Grinnell, 1963; Noctilio leporinus, Wenstrup, 1984; Lasiurus borealis, Obrist and Wenstrup, 1998; Pipistrellus abramus, Simmons et al., 2015; Eptesicus fuscus, Simmons et al., 2016 and Phyllostomus discolor, Linnenschmidt and Wiegrebe, 2019), but the EFR has not been recorded in bats, so far.

Here we describe the EFR of juvenile Phyllostomus discolor at ages between $\mathrm{p} 8$ and p72. EFRs of individual animals showed a considerable amount of variability. In two out of three animals, the EFR developed with age, showing an increase in peak amplitude within the ABRs modulation spectra. Age related changes were stronger for high modulation frequencies $(87-130 \mathrm{~Hz})$ compared to lower modulation frequencies $(11-58 \mathrm{~Hz})$.

\section{Methods}

\subsection{Animals}

All the experiments complied with the principles of laboratory animal care and were conducted following the regulations of the current version of the German Law on Animal Protection (approval ROB-55.2-2532.Vet_02-17-218, Regierung von Oberbayern). The bats (Phyllostomus discolor; two juvenile females, one juvenile male, aged p8 on the first day of experiments) originated from a breeding colony situated in the Department Biology II of the Ludwig-Maximilian University of Munich. For experiments, animals were kept together with their mothers under semi-natural conditions ( 12 h day/12 h night cycle, $65 \%-70 \%$ relative humidity, $28{ }^{\circ} \mathrm{C}$ ) with free access to food and water.

\subsection{Anaesthesia}

The bats were anaesthetized using a combination of medetomidine (Dorbene ${ }^{\circledR}$, Zoetis), midazolam (Dormicum ${ }^{\circledR}$, Hoffmann-La Roche) and fentanyl (Fentadon ${ }^{\circledR}$, Albrecht) at a dosage of $0.4,4.0$ and $0.04 \mu \mathrm{g} / \mathrm{g}$ body weight, respectively.

The anaesthesia was antagonized with a mixture of atipamezole (Alzane ${ }^{\circledR}$, Novartis), flumazenil (Flumazenil, Hexal) and naloxone (Naloxon-ratiopharm ${ }^{\circledR}$, Ratiopharm), which was injected subcutaneously (2.5, 0.5 and $1.2 \mu \mathrm{g} / \mathrm{g}$ body weight, respectively). During the experiments, the eyes of the animals were covered with a vitamin A cream (VitA POS $®$, Ursapharm) to prevent them from drying, whereas the puncture wounds of the ABR electrodes were treated with panthenol (Bepanten ${ }^{\circledR}$, Bayer).

\subsection{Acoustic box setup}

The setup used during the experiments is a close copy of the setup used by Linnenschmidt and Wiegrebe (2019). All recordings were made in a small, heavily sound-attenuated box (outside dimensions $34 \times 34 \times 34 \mathrm{~cm}$ ) constructed from veneer plywood $(4 \mathrm{~cm}$ thick) and covered with a removable acrylic glass lid ( $2.5 \mathrm{~cm}$ thick). The walls and floor were covered in $2 \mathrm{~cm}$ acoustic foam to dampen unwanted reflections of the stimuli. During the experiments, the bats were placed on a styrene foam block on axis with the loudspeaker. Two heating pads $(11.0 \times 7.7 \mathrm{~cm}, 12 \mathrm{~V}, 12 \mathrm{~W}$, thermo Flächenheizungs $\mathrm{GmbH}$, Rohrbach, Germany) were located on either side of the animal. These were supplied by an external power supply (Voltcraft LPS 1153, Conrad Electronics SE, Hirschau, Germany). The temperature was monitored by an analogue thermometer tucked under the wing right next to the body of the bat and maintained at $37^{\circ} \mathrm{C}$ air-temperature.

\subsection{Acoustic stimulation}

In order to measure the ABR properties to sinusoidally amplitude modulated (SAM) sounds, we followed the procedure used by Linnenschmidt and Wiegrebe (2019). First, the response threshold was established by presenting short, broadband $(4-96 \mathrm{kHz}$, flat power spectrum, 256 repetitions) click stimuli to the animals. The stimuli were presented at nine different sound pressure levels (SPL) ranging from 40 to $120 \mathrm{~dB}$ (peak-equivalent, re $20 \mu \mathrm{Pa}$ ) in $10 \mathrm{~dB}$ steps with every second stimulus played phase-inverted.

To evaluate the envelope following properties of the auditory brainstem a $200 \mathrm{~ms}$ frozen noise carrier $(2-95 \mathrm{kHz}$, flat power spectrum) was modulated with seven different modulation sinusoids logarithmically spaced from 11 to $130 \mathrm{~Hz}$. This range of modulation frequencies (MF) is most prominent in the temporal envelope of communication sound of $P$. discolor (Hörpel and Firzlaff, 2019).The modulation depth was $100 \%$. The modulation frequencies were each randomly presented 256 times (every second stimulus was phase-inverted) at a level of $80 \mathrm{~dB}$ RMS ( $99 \mathrm{~dB}$ peSPL). The repetition rate was approximately $1.2 \mathrm{~Hz}$.

All acoustic stimuli were computer-generated (Matlab $\AA$, 2018b; Mathworks, Natick, MA, USA), digital-analogue converted (Fireface 400, RME, Haimhausen, Germany; sampling rate $192 \mathrm{kHz}$ ), amplified (AX-396, Yamaha Music Foundation, Tokyo, Japan) and presented via free-field loudspeaker (R2904/700000, Scan-Speak, Videbæk, Denmark). The loudspeaker had been calibrated for linear frequency response between $1 \mathrm{kHz}$ and $96 \mathrm{kHz}$ and was positioned on the midline roughly $8 \mathrm{~cm}$ from the bats ears.

\subsection{Electrophysiological recordings}

To record the auditory brainstem responses, the setup was connected via small alligator clips to three subdermal electrodes (clipped $0.3 \times 12 \mathrm{~mm}$ hypodermic needles, Henry Schein Inc., Melville, USA), which were pushed through the skin of the animal. The recording electrode was placed centrally at the base of the skull above the brainstem (Nape) while the reference electrode was placed centrally on top of the skull at the height of the foremost edge of the ears (Vertex). A ground electrode was inserted into the tissue caudally of the base of the right ear. The signals were initially amplified by 20 dB (DAM80, World Precision Instruments, Sarasota, USA) with the amplifier set to its widest filter settings $(0.1 \mathrm{~Hz}-10 \mathrm{kHz}$ band-pass filter). A HumBug (Quest Scientific, North Vancouver, Canada) was used to remove noise from the power mains, but did not affect the ABR signal. The signals were then analog-to-digitally converted (Fireface 400; $192 \mathrm{kHz}$ sampling rate), additionally amplified by $10 \mathrm{~dB}$ and down-sampled 10 times 
to reduce the amount of required storage. Then, each of the 256 repetitions per stimulus and sound level was saved into one combined file for offline analysis.

\subsection{Data analysis}

In order to establish click evoked ABRs waveforms recorded from the 256 stimuli, the repetitions were averaged and filtered ( $100 \mathrm{~Hz}-3000 \mathrm{~Hz}$ band-pass). To determine click evoked ABR SPL thresholds, we utilized a bootstrap analysis as described in Lv et al. (2007) and used by Linnenschmidt and Wiegrebe (2019). An analysis window of $6.8 \mathrm{~ms}$ ( $0.2 \mathrm{~ms}-7 \mathrm{~ms}$ after stimulus onset) was chosen. The bootstrap method is based on repeatedly drawing random samples of that window size (with replacement) from the original data (500 repetitions). It is then assessed in which percentage of cases the RMS of the resampled waveform exceeds the RMS of the original waveform. An ABR is considered significant when more than $99 \%$ of resampled waveforms have a lower RMS than the original waveform (Linnenschmidt and Wiegrebe, 2019; Lv et al., 2007).

ABRs evoked by SAM-noise were also established by averaging the recorded waveforms to 256 stimulus repetitions (but no filtering occurred). We then analyzed the modulation spectra of the averaged waveforms. In detail, we calculated the FFT (Fast Fourier transform, Matlab ${ }^{\circledR}$ ) of the waveforms Hilbert envelope. EFRs were quantified by the height of the peak in the modulation spectra within the MF frequency range. However, the ABR amplitude (and thus the height of the envelope modulation spectra peaks) may not only be influenced by undesired factors like small differences in the positions of the recording electrode, but also by growth of the animal during post-natal development (Linnenschmidt and Wiegrebe, 2019; Walsh et al., 1986). Therefore, as a check, we also analyzed the EFR based on root mean square (RMS)-normalized average waveforms.

\section{Results}

In total, we recorded ABRs on 24 different occasions ( 3 animals, 8 recordings spread over 3 months from day p8-p72).

\subsection{Development of click evoked ABRs}

Click evoked ABRs with stable waveforms with multiple positive peaks were obtained for all animals. Examples of click evoked ABRs from two different bats are shown in Fig. 1a,b,d,e for different presentation levels ranging from $40 \mathrm{~dB}$ peSPL up to $120 \mathrm{~dB}$ peSPL. For the highest presentation level, the waveform typically consisted of 7 distinguishable waves and one pre-potential. The pre-potential occurred ca. $0.8 \mathrm{~ms}$ after stimulus onset, while the dominant waves $\mathrm{I}-\mathrm{V}$ followed between 1.7 and $5 \mathrm{~ms}$. Waves I and II were partly merged, i.e. they were protruding from each others rising or falling flanks. The same was observed for waves III-V. This waveform pattern was most prominent in early stages of the juvenile development (Fig. 1 a,d), and became more ambiguous in older bats (Fig. 1b,e). During development of the juveniles, the overall RMS amplitude of the click evoked ABRs generally decreased (Fig. 1c,f) for higher presentation levels, although this trend was not strictly linear in all three bats. For example, in Bat 1 (Fig. 1c) the ABR RMS amplitude resulting from the loudest presentation level increased slightly until p15 before a strong decrease was observed over the following period of juvenile development up to $\mathrm{p} 69$.

In Bat 3 click evoked ABR waveforms showed less distinguishable waves (Supplement S1). Contrarily to Bat 1 and 2, waves I and II were barely indistinguishable from each other and wave VII was missing.
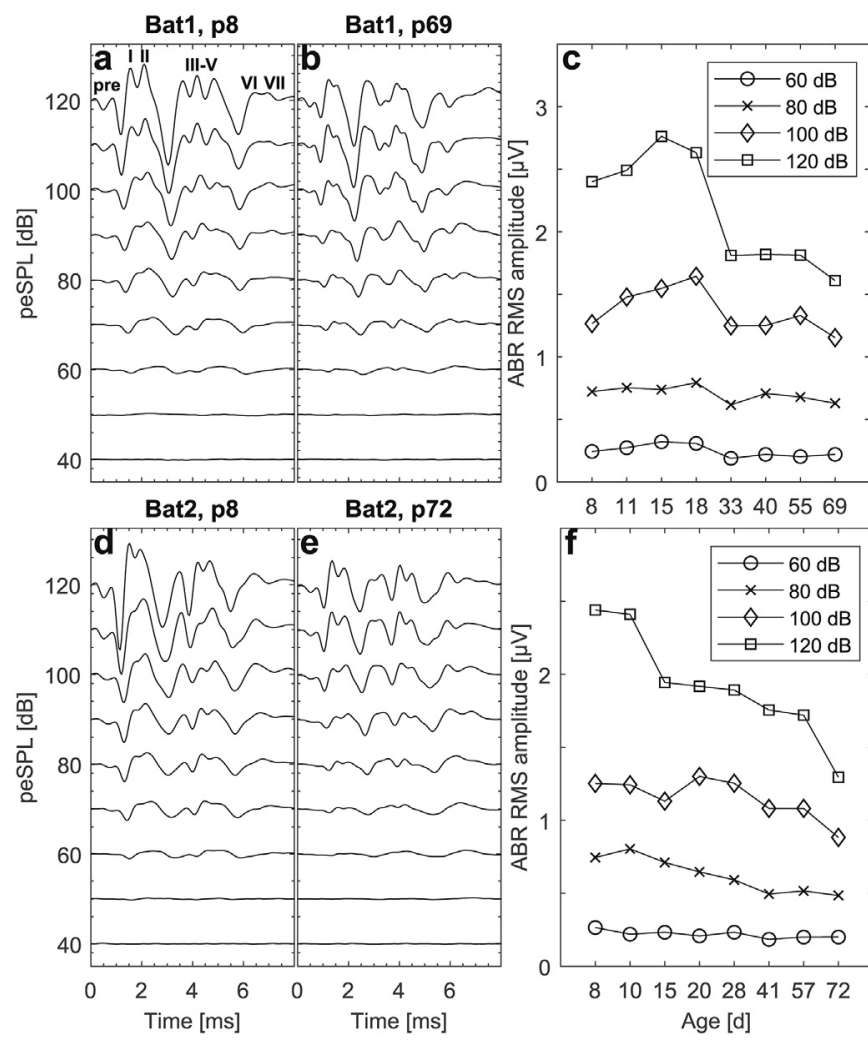

Fig. 1. Influence of stimulus level and age on click evoked ABR waveforms in P. discolor in Bat 1 (a, b) and Bat 2 (d, e). c, f. Summary data for ABR RMS amplitudes for all recording dates for Bat 1 and Bat 2, respectively. Analysis window for RMS calculation was from 0.2 to $7 \mathrm{~ms}$.

Using click stimuli, significant ABRs could be evoked for presentation levels as low as $50 \mathrm{~dB}$ SPL in all three animals. This threshold did not change during development (Fig. 1a,b,d,e; S1), but fluctuated between $50 \mathrm{~dB}$ SPL and $60 \mathrm{~dB}$ SPL in all three animals.

\subsection{Envelope following response}

When comparing Fig. 1 with Fig. 2, the differences in fine structure between the ABR waveforms resulting from click stimulation or amplitude modulated noise stimulation become apparent: While the ABR waveform evoked by clicks is of limited duration ( $\sim 7 \mathrm{~ms}$, see Fig. 1a,b,d,e), the SAM evoked waveform is longer and more complex. Moreover, single waveform components within the click ABR waveform get less distinguishable and the overall RMS amplitude decreases during development (see Fig. 1a,b,d,e). Contrarily, age dependent changes in the waveform structure visible for the SAM-noise ABR waveform show the opposite trend.

Fig. 2 (left column) shows a $90 \mathrm{~ms}$ excerpt (i.e. one modulation cycle), of a SAM-noise evoked ABR waveform for a modulation frequency of $11 \mathrm{~Hz}$, with the envelope of one modulation cycle of the stimulus (frozen noise) shown in the top panel. As the stimulus was frozen noise, the envelopes are not smooth, but show different numbers of prominent peaks within one modulation period. This is somewhat reflected in the evoked EFRs which accordingly also show a different structure of response peaks in one modulation cycle. For a lower modulation frequency, the number of structural peaks within one modulation period is of course higher than for a higher modulation frequency. At an early age ( 88$)$, no prominent waveform component is visible throughout the whole modulation 

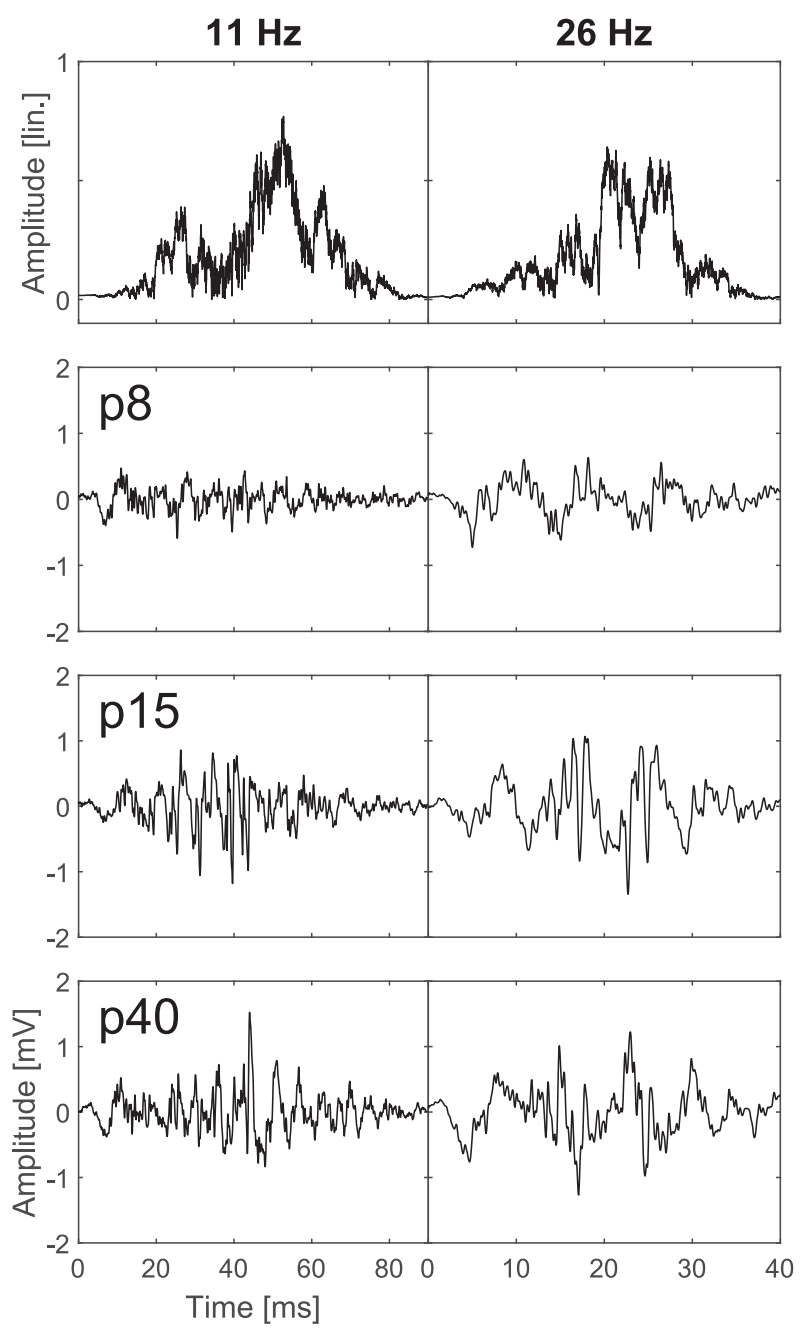

Fig. 2. Development of SAM noise evoked ABR waveforms for two MFs in Bat 1. In the top panel, the envelope of one modulation cycle of the respective stimulus is plotted. The waveforms corresponding to only one modulation cycle are shown in the lower three panels. Note different time scales of the abscissa of the left column and the right column.

cycle. For recordings on later days of juvenile development, large waveform components appear around the middle of the modulation cycle i.e. when the modulated stimulus reaches its peakamplitude. The same can be observed for the SAM evoked ABR waveform for a modulation frequency of $26 \mathrm{~Hz}$ (Fig. 2, right column).

\subsection{Development of the EFR}

Fig. 3 illustrates the development of EFRs in Bat 1 for the seven different modulation frequencies. The ABR waveforms (Fig. 3, row 1 to 3) show changes in both shape and structure with increasing age of the juvenile bat. Typically, the waveforms show more distinct peaks in the responses to single modulation cycles and thus the envelope becomes more distinct for older bats. Additionally, RMS amplitude values of the waveform typically increase during postnatal development of the bats (Fig. 4) at least for higher MFs. Consecutively, these effects lead to an increase in the peak magnitude of the envelope modulation spectra in the frequency band corresponding to the modulation frequency (Fig. 5).
The development of the peak magnitude of the modulation frequency's envelope spectra in Bat 1 is shown in Fig. 3 (bottom row). Although peak magnitudes show some degree of fluctuations, for most modulation frequencies, the peak magnitude increases with age until it reaches a plateau. An exception to this trend can be seen for the modulation frequencies of 17 and $26 \mathrm{~Hz}$, where the peak magnitude constantly decreases until p69 after a preliminary increase between $\mathrm{p} 11$ and $\mathrm{p} 15$. The peak magnitude values reached during the plateau-phase are in the same range for all MFs while the peak magnitude at $\mathrm{p} 8$ is lower for the highest MF $(130 \mathrm{~Hz})$.

The same developmental, age-dependent EFR pattern can be seen in the ABR measurements for Bat 2 (Fig. S2), with the exception, that for the MF of $130 \mathrm{~Hz}$, peak magnitude values do not reach the same level reached for other MFs. In Bat 3, peak magnitudes of envelope modulation spectra for MF below $87 \mathrm{~Hz}$ remain at about the same level over the whole period of measurements (Fig. S3). Only for the MFs of 87 and $130 \mathrm{~Hz}$ an increase in peak magnitude is seen during juvenile development, which is strong for $87 \mathrm{~Hz}$ but only weak for $130 \mathrm{~Hz}$.

In some cases, the ABR RMS amplitude displayed fluctuations over developmental time (see Fig. 4). We analyzed the EFR based on RMS-normalized averaged waveforms in order to guarantee that the nature of above-mentioned changes in the EFR were due to differences in its temporal fine structure and not only due to an increased ABR RMS amplitude.

The results show that the above-described age-dependent EFR pattern is also observable for normalized waveforms (Fig. 6 and Supplement S4). Thus, the fine structure of the SAM-noise evoked ABR waveform contributes substantially to the peak magnitude of envelope modulation spectra and to the maturation of the EFR, consequently.

\subsection{Time course and amount of EFR maturation}

As can be seen in Fig. 3, the chronological progression of the ERF maturation was different for the various MFs. In Bat 1, the increase of the peak magnitude of envelope modulation spectra reached a plateau on p15 for MFs up to $58 \mathrm{~Hz}$. For higher MFs ( 87 and $130 \mathrm{~Hz}$ ), the plateau-phase was reached later in time on p33. Differences between the different MFs can also been seen when comparing the range by which the peak magnitudes of envelope modulation spectra increased from the first measurement to the last recording day (Fig. 7, dark grey bars). For high MFs (87 and $130 \mathrm{~Hz}$ ) an overall increase of 24 and $30 \mathrm{~dB}$, respectively, was observed, whereas for lower MFs (11-58 Hz) peak magnitudes did not increase more than $18 \mathrm{~dB}$. Therefore, the EFR in Bat 1 seems to be already stronger developed in early stages of the juvenile development for lower MFs compared to higher MFs.

In Bat 2, the same trend can be observed (Fig. 7, medium grey bars): Except for the MF of $38 \mathrm{~Hz}$ (16 dB peak magnitude), the peak magnitude changes for lower MFs was lower (range: -1 to $5 \mathrm{~dB}$ ) than for the two highest MFs ( 25 and $10 \mathrm{~dB}$, respectively). In Bat 2, the plateau-phase of peak magnitudes was reached at p20 for all MFs (Fig. S2).

The EFR to MFs up to $58 \mathrm{~Hz}$ showed little to no maturation in Bat 3 (Fig. 7, light grey bars). Consequently, the increase of peak magnitudes over time was low to non-existent and fluctuated in a range of -6 to $+3 \mathrm{~dB}$. Contrarily, MFs of 87 and $130 \mathrm{~Hz}$ showed an overall increase in the peak magnitude of the modulation spectrum (18 and $7 \mathrm{~dB}$, respectively), which, however, was still less when compared to the increase observed in Bat 1 and Bat 2. In summary, at least in two animals, the maturation of the EFR was stronger and took longer for modulation frequencies above $87 \mathrm{~Hz}$ than for lower MFs, for which the EFR could be quite robust already at early stages of the post-natal development (from $\mathrm{p} 8$ on). 


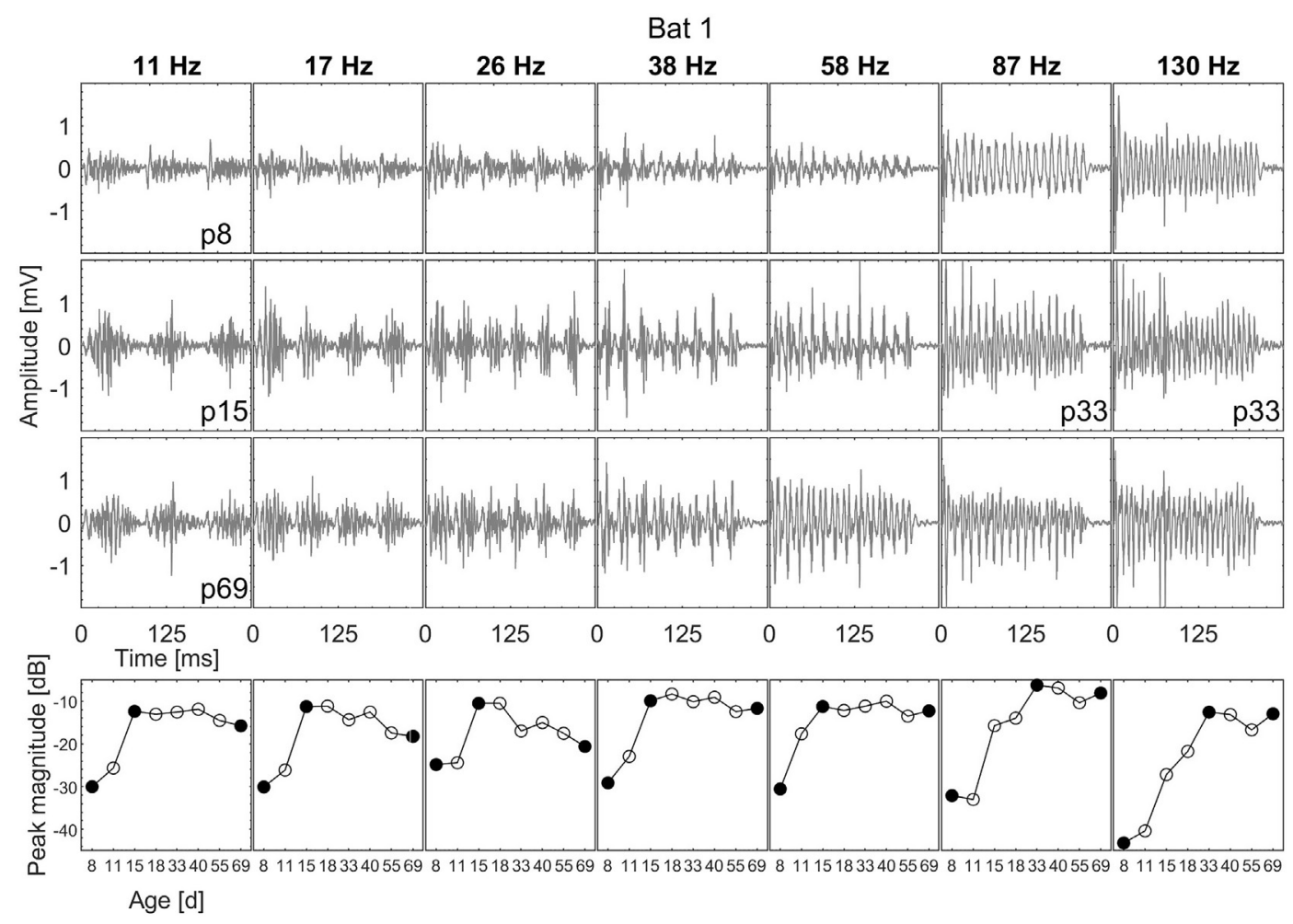

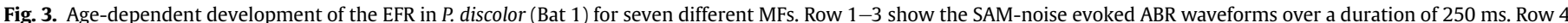
shows the peak magnitude of the waveform-envelope modulation spectra. Black dots represent the postnatal days on which the waveforms shown in row1-3 were recorded.

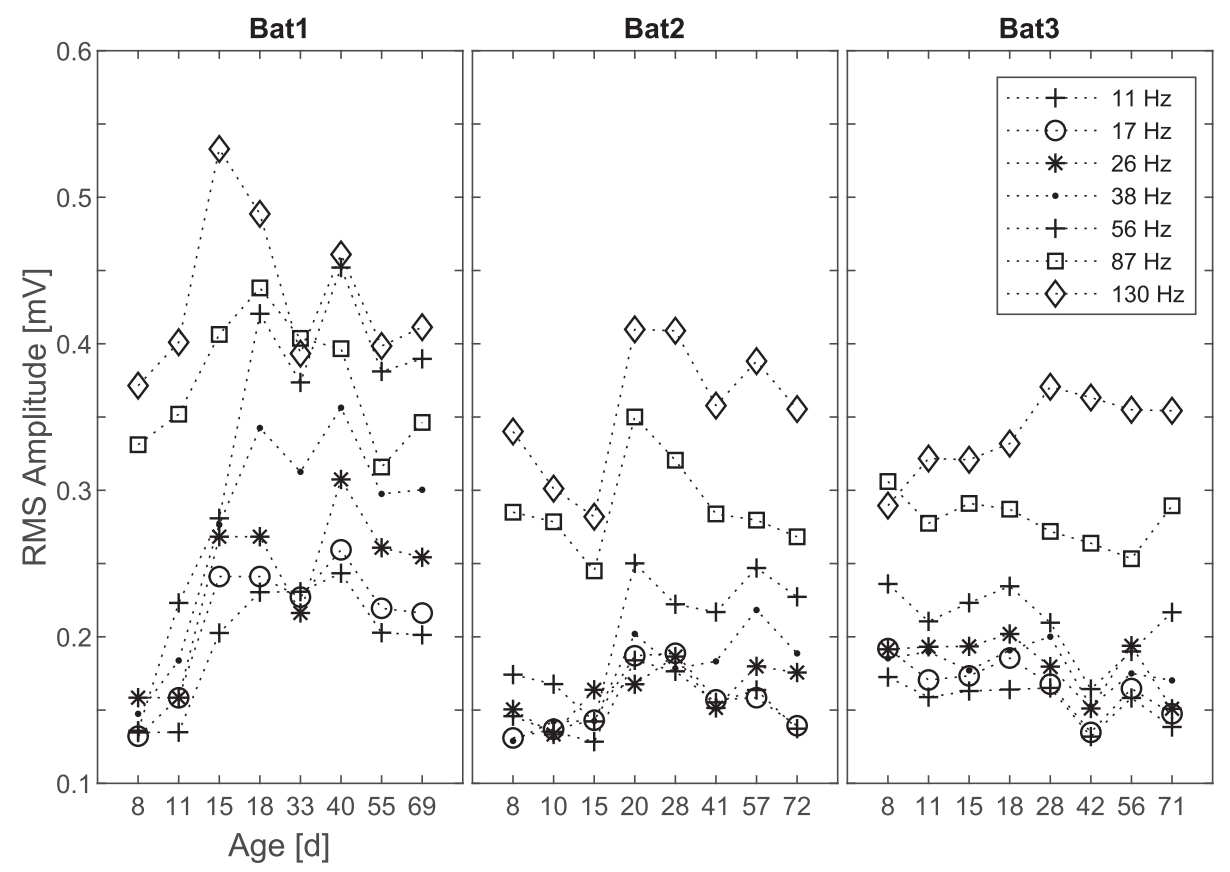

Fig. 4. Age-dependent development of the RMS amplitude of the SAM-noise evoked ABR waveforms.

\section{Discussion}

We measured EFRs during juvenile development of three $P$. discolor pups. EFRs were already present on p8. In two out of three animals, peak magnitude of envelope modulation spectra of SAM evoked ABRs increased consistently during development for most MFs, whereas the maturation of the EFR was slower for higher MFs. 

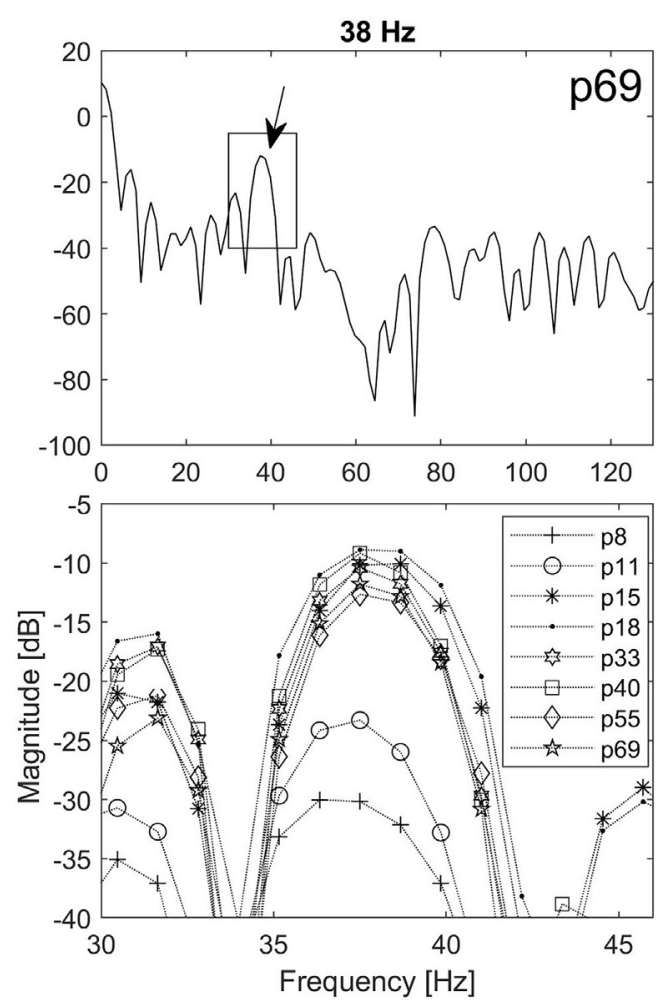

Fig. 5. Top: Modulation spectrum for the $\mathrm{SAM}(\mathrm{MF}=38 \mathrm{~Hz})$ evoked EFR recorded on p69 in Bat 1. The resulting spectral peak at $38 \mathrm{~Hz}$ is indicated by an arrow. Bottom: Age-dependent development of this spectral peak.

\subsection{Waveform and development of click evoked ABRs}

We measured click evoked ABRs to assess basic hearing abilities of the pups and as a quality check for recording conditions. The overall waveforms and duration of click evoked ABRs recorded in the present study were quite similar to those recorded by Linnenschmidt and Wiegrebe (2019) in the same species and up to seven waves were identifiable. Slight differences in the positioning of the electrodes might have contributed to the small differences observed in our recordings (e.g. ABR Wave II was as strong as Wave I) when compared with the results of Linnenschmidt and Wiegrebe (2019). Hearing thresholds established by click ABR fluctuated between 50 and $60 \mathrm{~dB}$, which is $10-20 \mathrm{~dB}$ lower than measured by Linnenschmidt and Wiegrebe (2019), but still in a comparable range. Again, these differences might be due to slight differences in the recording set up e.g. in the positioning of the bats relative to the speaker. . Click ABR amplitudes typically decreased during juvenile development of the pups, however, as discussed in Linnenschmidt and Wiegrebe (2019), this effect is most probably due to growth of the head and attached muscles by which the electrodes become located in larger distance to the brain.

\subsection{Development of the SAM-noise evoked ABR waveform and the EFR}

As a trend, the RMS amplitude of the EFR increased with age at least for higher MFs. This finding falls in line with reported studies in rats and humans (e.g. Nodarse et al., 2012; Prado-Gutierrez et al., 2012) and can most likely be attributed to an increasing number of sensitive neurons and/or increasing neuronal temporal firing precision during post-natal development (Venkataraman and Bartlett, 2014).

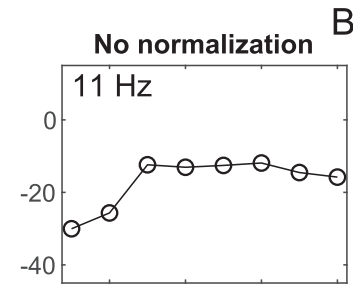

Bat 1
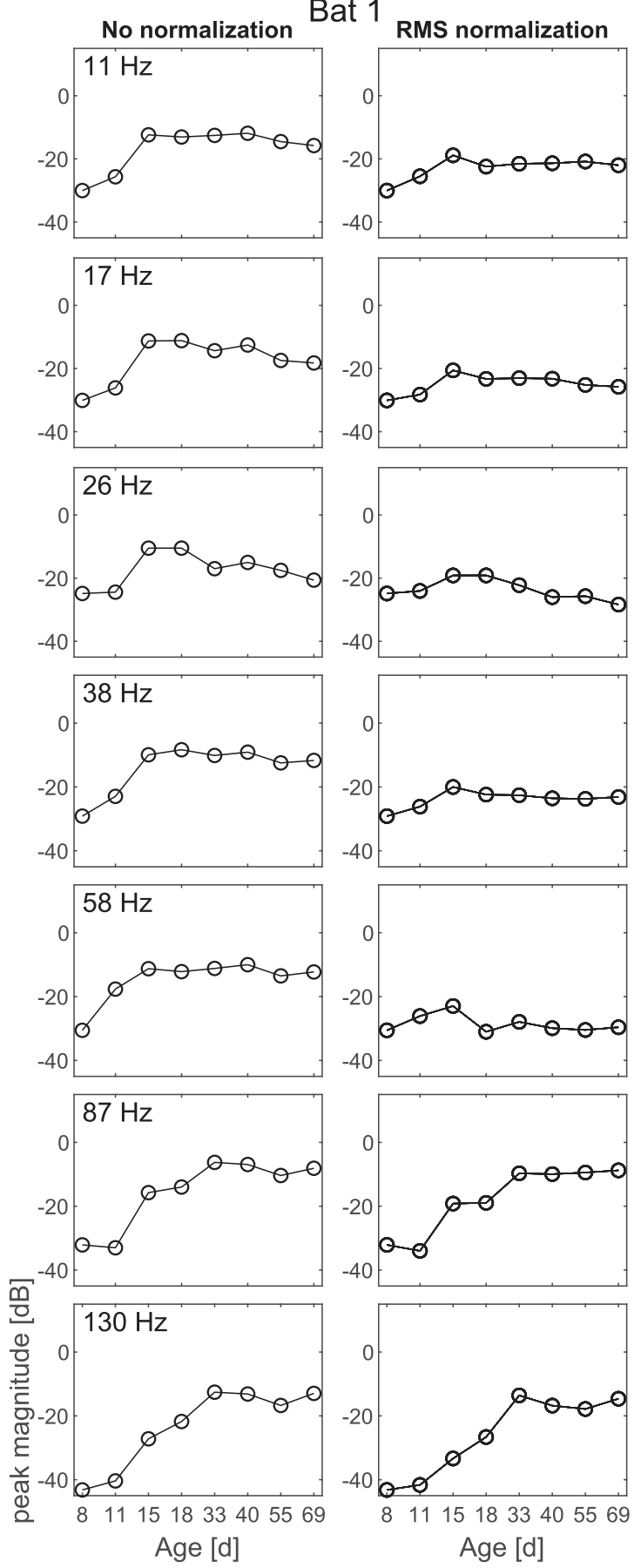

Fig. 6. Comparison of the age dependent development of the EFR in Bat 1 for nonnormalized (left column, replotted from Fig. 3) and RMS-normalized SAM-noise evoked ABR waveforms (right column).

The increasing "peakyness" shown in Fig. 2 and described in 3.2 has also been shown in humans (Rance et al., 2006). Similar to our findings with click evoked ABRs, the threshold level of tone-burst evoked ABRs did not change and strong, "peaky" waves in the ABR responses emerged during maturation of the child.

In our study, the observed shape of SAM-noise evoked ABR waveforms to higher MFs corresponded to the waveforms shown in Venkataraman and Bartlett (2014). The observed changes in the ABR waveform shape might be explained by maturation of nuclei of 


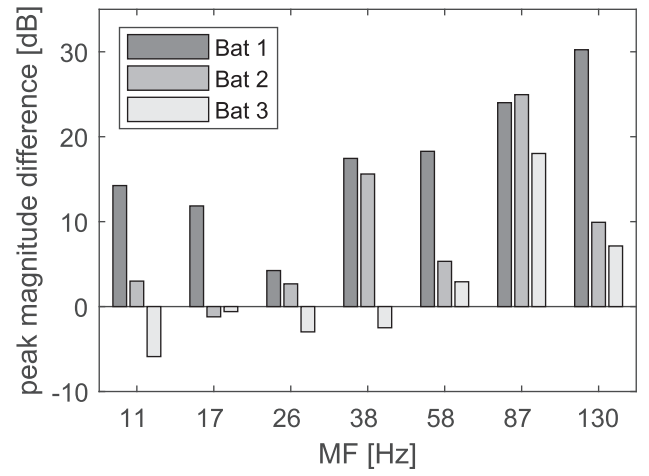

Fig. 7. Quantification of the MF dependent changes of the EFR during maturation for all three bats.

Changes in peak magnitude were calculated between the p8 and p69 (Bat 1), p72 (Bat 2) and p71 (Bat 3).

the ascending auditory pathway involved in amplitude modulation coding.

As a general trend, the fidelity by which ABR could follow noise envelope modulations increased during post-natal development in $P$. discolor pups. This was especially the case for the range of higher modulation frequencies tested in our study. Our findings corroborate earlier studies showing increasing best MFs to SAM stimuli (both tonal and noise carriers) and click-trains during the juvenile development of EFRs in rats (Prado-Gutierrez et al., 2012; Venkataraman and Bartlett, 2013) and for neuronal spike activity in the inferior colliculus (IC) of gerbils (Heil et al., 1995) and the auditory cortex of cats (Eggermont, 1993, 1991). Responsiveness to higher click-rates also increased in the cochlear nerve and the IC in mice (Sanes and Constantine-Paton, 1985).

The above-described development-dependent changes can be contributed to maturational processes of the cochlea (Johnson et al., 2005) and the ascending auditory pathway (e.g. increasing number of action potentials (AP) in stellate cells or decreasing AP duration in bushy cells (Müller et al., 2019)). Additionally, the myelination of axons and maturation of mechanisms underlying synaptic transmission also add to said changes (Brenowitz and Trussell, 2001; Müller et al., 2019; Prado-Gutierrez et al., 2012; Shu Hui Wu and Oertel, 1987; Venkataraman and Bartlett, 2013). In cats, the onset of myelination occurred at p5 and reached a rate of $70 \%$ by p10 (Ryugo et al., 2006). The endbulbs of Held undergo a reorganization into smaller compartments while increasing the volume fraction of the mitochondria and the synaptic vesicle density during the postnatal development of the animal. These changes ensure time-precise synaptic transmissions with short delay, which should be important for the encoding of high modulation frequencies (Ryugo et al., 2006).

The strong individual differences observed in the EFRs especially in Bat 3 might be contributed to differences in the electrode positioning, which is extremely intricate in bat pups of this young due to the small head size. This is supported by differences in the wave components of the click evoked ABRs (compare Figs. 1 and S1). Furthermore, we can of course not exclude developmental deficits of the auditory pathway in individual animals, although physically all three pups developed and behaved similarly.

In gerbils, a decrease of magnitude in the modulation spectrum with increasing modulation frequency has already been described (Dolphin and Mountain, 1992). In the range of 20-100 Hz modulation frequency, the peak magnitude fluctuated by $\pm 3-4 \mathrm{~dB}$, whereas for modulation frequencies greater than $100 \mathrm{~Hz}$ a decrease of 7-20 dB was observed. Similar findings have been reported for Fisher-344 rats, were strong decreases in the peak magnitude in the modulation spectrum were observed for modulation frequencies greater than $100 \mathrm{~Hz}$ (Parthasarathy et al., 2014). In our data, two out of three bats also displayed decreases in the peak magnitude of the modulation spectrum for frequencies larger than $100 \mathrm{~Hz}$ (compare Figs. S2 and S3, bottom row). However, in Bat 1 such a decrease was not observable. Therefore, it is not clear if this decrease might still be an effect of individual maturation, even though all three animals were of comparable age when measured.

As described by Dolphin et al. (1995), cetacean species do not show a drop-off in magnitude at such low modulation frequencies. The false killer whale Pseudorca crassidens showed no strong dropoff of the magnitude in the modulation spectrum for modulation frequencies of up to $1300 \mathrm{~Hz}$, although a smaller drop-off occurred between 300 and $450 \mathrm{~Hz}$. Similar findings were reported for the bottlenosed dolphin Tursiops truncatus, where the strong drop-off was shown for frequencies larger than $1500 \mathrm{~Hz}$, with a slip dip in magnitude between 250 and $400 \mathrm{~Hz}$ modulation frequency. Therefore, there might be a preadaptation for high-fidelity temporal processing in echo locating animals, which might explain the differences in cut-off modulation frequency compared to rats and gerbils.

\subsection{Functional implication for vocal development and species- specific communication}

The here described developmental changes in the EFR (i.e. the improved responsiveness of the auditory brainstem to temporal modulation of sound envelope), although only observed in two of three juveniles of the bat $P$. discolor, might have (if generally applied) consequences on the development of vocal communication of this species. New born P. discolor pups reared without their mothers showed an altered structure (i.e. a reduced amount of temporal modulations) of the contact calls, typically used to communicate with their mothers (Esser and Schmidt, 1989). Other studies reported changes in fundamental frequency of the vocal communication repertoire of juvenile bats, which had been reared in isolation from adult conspecifics (Prat et al., 2015).

According to Esser and Schmidt (1989), communication between pups and mothers is, of course, bidirectional. The mother emit directive calls with resemble the contact/isolation calls emitted by the pups in their basic spectro-temporal structure. As described by Esser and Schmidt, the structure of the directive call influences the development of the isolation calls. Directive calls typically show frequency modulation, but also lesser degrees of amplitude modulation (Lattenkamp et al., 2019). However, in a natural social environment, pups also experience all other types of communication calls emitted by other group members. These include strongly amplitude modulated calls e.g. aggression calls (Hörpel and Firzlaff, 2019; Lattenkamp et al., 2019). Especially for vocal learning, auditory feedback is important to produce sounds that match a heard sound template (Brainard and Doupe, 2000; Nordeen and Nordeen, 1992; Tyack, 2016). The maturation period of the auditory system of $P$. discolor, and, regarding the prominent amount of temporal modulations in the communication calls of this species, especially the maturation of the EFR, therefore constitutes an important period during which the development of the vocal repertoire is accomplished. The fact, that at least for lower modulation frequencies the EFR can already be present (although not fully developed) at early ages of juvenile development (p8) would further support a possible role of processing of temporal modulation in mother infant communication. 


\section{Grants}

This work was supported by Human Frontier Science Program (France)-Grant (RGP0058) to U. Firzlaff.

\section{Declaration of competing interest}

The authors declare no competing financial interests.

\section{CRediT authorship contribution statement}

Stephen Gareth Hörpel: Conceptualization, Methodology, Software, Investigation, Writing - original draft, Visualization. Uwe Firzlaff: Conceptualization, Methodology, Software, Writing original draft, Visualization, Supervision, Funding acquisition.

\section{Acknowledgements}

We thank Lutz Wiegrebe for providing the experimental animals, his comments and input into the analysis. We would also like to thank Lutz Kettler for his comments on an earlier version of the manuscript.

\section{Appendix A. Supplementary data}

Supplementary data to this article can be found online at https://doi.org/10.1016/j.heares.2020.107904.

Abbreviations
$\begin{array}{ll}\text { ABR } & \text { auditory brainstem response } \\ \text { EFR } & \text { envelope following response } \\ \text { FFT } & \text { fast Fourier transformation } \\ \text { ITD } & \text { interaural time differences } \\ \text { RMS } & \text { root mean square } \\ \text { SAM } & \text { sinusoidally amplitude modulated } \\ \text { (pe)SPL } & \text { (peak equivalent) sound pressure level re } 20 \mu \mathrm{Pa}\end{array}$

\section{References}

Bohn, K.M., Schmidt-French, B., Ma, S.T., Pollak, G.D., 2008. Syllable acoustics, temporal patterns, and call composition vary with behavioral context in Mexican free-tailed bats. J. Acoust. Soc. Am. 124, 1838-1848. https://doi.org/ $10.1121 / 1.2953314$.

Boughman, J.W., 1998. Vocal learning by greater spear-nosed bats. Proc. R. Soc. B Biol. Sci. 265, 227-233. https://doi.org/10.1098/rspb.1998.0286.

Brainard, M.S., Doupe, A.J., 2000. Auditory feedback in learning and maintenance of vocal behaviour. Nat. Rev. Neurosci. 1, 31-40. https://doi.org/10.1038/35036205.

Brenowitz, S., Trussell, L.O., 2001. Maturation of synaptic transmission at end-bulb synapses of the cochlear nucleus. J. Neurosci. 21, 9487-9498.

Brown, P.E., Grinnell, A.D., Harrison, J.B., 1978. The development of hearing in the pallid bat, antrozous pallidus. J. Comp. Physiol. 126, 169-182. https://doi.org/ 10.1007/BF00666371.

Burkard, R.F., Eggermont, J.J., Don, M., 2007. Auditory Evoked Potentials: Basic Principles and Clinical Application. Lipincott Williams \& Wilkins.

Dimitrijevic, A., Alsamri, J., John, M.S., Purcell, D., George, S., Zeng, F.-G., 2016. Human envelope following responses to amplitude modulation: effects of aging and modulation depth. Ear Hear. 37.

Dolphin, W.F., Mountain, D.C., 1992. The envelope following response: scalp potentials elicited in the Mongolian gerbil using sinusoidally AM acoustic signals. Hear. Res. 58, 70-78. https://doi.org/10.1016/0378-5955(92)90010-K.

Dolphin, W.F., Au, W.W.L., Nachtigall, P.E., Pawloski, J., 1995. Modulation rate transfer functions to low-frequency carriers in three species of cetaceans. J. Comp. Physiol. 177, 235-245. https://doi.org/10.1007/BF00225102.

Draganova, R., Schollbach, A., Schleger, F., Braendle, J., Brucker, S., Abele, H., Kagan, K.O., Wallwiener, D., Fritsche, A., Eswaran, H., Preissl, H., 2018. Fetal auditory evoked responses to onset of amplitude modulated sounds. A fetal magnetoencephalography (fMEG) study. Hear. Res. 363, 70-77. https://doi.org/ 10.1016/j.heares.2018.03.005.

Eggermont, J.J., 1991. Rate and synchronization measures of periodicity coding in cat primary auditory cortex. Hear. Res. 56, 153-167. https://doi.org/10.1016/03785955(91)90165-6.
Eggermont, J.J., 1993. Differential effects of age on click-rate and amplitude modulation-frequency coding in primary auditory cortex of the cat. Hear. Res. 65, 175-192. https://doi.org/10.1016/0378-5955(93)90212-J.

Esser, K.-H., Schmidt, U., 1989. Mother-infant communication in the lesser spearnosed bat Phyllostomus discolor (chiroptera, phyllostomidae) - evidence for acoustic learning. Ethology 82, 156-168. https://doi.org/10.1111/j.14390310.1989.tb00496.x.

Esser, K.-H., Schmidt, U., 1990. Behavioral auditory thresholds in neonate lesser spear-nosed bats. Phyllostomus discolor. Sci. Nat. 77, 292-294. https://doi.org/ 10.1007/BF01131230.

Gaucher, Q., Huetz, C., Gourévitch, B., Laudanski, J., Occelli, F., Edeline, J.M., 2013. How do auditory cortex neurons represent communication sounds? Hear. Res. 305, 102-112. https://doi.org/10.1016/j.heares.2013.03.011.

Grinnell, A.D., 1963. The neurophysiology of audition in bats: resistance to interference. J. Physiol. 167, 114-127.

Heil, P., Schulze, H., Langner, G., 1995. Ontogenetic development of periodicity coding in the inferior colliculus of the Mongolian. Gerbil. Audit. Neurosci. 1, 363-383.

Hörpel, S.G., Firzlaff, U., 2019. Processing of fast amplitude modulations in bat auditory cortex matches communication call-specific sound features. J. Neurophysiol. 121 https://doi.org/10.1152/jn.00748.2018.

Johnson, S.L., Marcotti, W., Kros, C.J., 2005. Increase in efficiency and reduction in $\mathrm{Ca} 2+$ dependence of exocytosis during development of mouse inner hair cells. J. Physiol. 563, 177-191. https://doi.org/10.1113/jphysiol.2004.074740.

Khurana, S., Liu, Z., Lewis, A.S., Rosa, K., Chetkovich, D., Golding, N.L., 2012. An essential role for modulation of hyperpolarization-activated current in the development of binaural temporal precision. J. Neurosci. 32, 2814-2823. https://doi.org/10.1523/JNEUROSCI.3882-11.2012.

Knörnschild, M., 2014. Vocal production learning in bats. Curr. Opin. Neurobiol. 28 80-85. https://doi.org/10.1016/j.conb.2014.06.014.

Knörnschild, M., Feifel, M., Kalko, E.K.V., 2014. Male courtship displays and vocal communication in the polygynous bat Carollia perspicillata. Behaviour 151, 781-798.

Kraus, H.J., Aulbach-Kraus, K., 1981. Morphological changes in the cochlea of the mouse after the onset of hearing. Hear. Res. 4, 89-102. https://doi.org/10.1016/ 0378-5955(81)90038-1.

Lattenkamp, E.Z., Shields, S.M., Schutte, M., Richter, J., Linnenschmidt, M. Vernes, S.C., Wiegrebe, L., 2019. The vocal repertoire of pale spear-nosed bats in a social roosting context. Front. Ecol. Evol. 7, 1-14. https://doi.org/10.3389/ fevo.2019.00116.

Linnenschmidt, M., Wiegrebe, L., 2019. Ontogeny of auditory brainstem responses in the bat, Phyllostomus discolor. Hear. Res. 373, 85-95. https://doi.org/10.1016/ j.heares.2018.12.010.

Linnenschmidt, M., Wahlberg, M., Damsgaard Hansen, J., 2013. The modulation rate transfer function of a harbour porpoise (Phocoena phocoena). J. Comp. Physiol. A 199, 115-126. https://doi.org/10.1007/s00359-012-0772-8. Neuroethol. Sensory, Neural, Behav. Physiol.

Lv, J., Simpson, D.M., Bell, S.L., 2007. Objective detection of evoked potentials using a bootstrap technique. Med. Eng. Phys. 29, 191-198. https://doi.org/10.1016/ j.medengphy.2006.03.001.

Magnusson, A.K., Kapfer, C., Grothe, B., Koch, U., 2005. Maturation of glycinergic inhibition in the gerbil medial superior olive after hearing onset. J. Physiol. 568, 497-512. https://doi.org/10.1113/jphysiol.2005.094763.

Mikaelian, D., Ruben, R.J., 1965. Development of hearing in the normal cba-j mouse: Correlation of physiological observations with behavioral responses and with cochlear anatomy. Acta Oto- laryngologica.

Moore, D.R., 1982. Late onset of hearing in the ferret. Brain Res. 253, 309-311. https://doi.org/10.1016/0006-8993(82)90698-9.

Moushegian, G., Rupert, A.L., Stillman, R.D., 1973. Scalp-recorded early responses in man to frequencies in the speech range. Electroencephalogr. Clin. Neurophysiol. 35, 665-667. https://doi.org/10.1016/0013-4694(73)90223-X.

Müller, M.K., Jovanovic, S., Keine, C., Radulovic, T., Rübsamen, R., Milenkovic, I., 2019. Functional development of principal neurons in the anteroventral cochlear nucleus extends beyond hearing onset. Front. Cell. Neurosci. 13, 1-21. https:// doi.org/10.3389/fncel.2019.00119.

Nodarse, E.M., Abalo, M.C.P., Fortuny, A.T., Hernández, M.V., Castellanos, A.L., 2012 Maturational changes in the human envelope-following responses. Acta Otorrinolaringol. 63, 258-264.

Nordeen, K.W., Nordeen, E.J., 1992. Auditory feedback is necessary for the maintenance of stereotyped song in adult zebra finches. Behav. Neural. Biol. 57, 58-66. https://doi.org/10.1016/0163-1047(92)90757-U.

Nourski, K.V., Brugge, J.F., 2011. Representation of temporal sound features in the human auditory cortex. Rev. Neurosci. 22, 187-203. https://doi.org/10.1515/ RNS.2011.016.

Obrist, M.K., Wenstrup, J.J., 1998. Hearing and hunting in red bats (Lasiurus borealis, Vespertilionidae): audiogram and ear properties. J. Exp. Biol. 201, $143-154$

Parthasarathy, A., Datta, J., Torres, J.A.L., Hopkins, C., Bartlett, E.L., 2014. Age-related changes in the relationship between auditory brainstem responses and envelope-following responses. JARO J. Assoc. Res. Otolaryngol. 15, 649-661. https://doi.org/10.1007/s10162-014-0460-1.

Prado-Gutierrez, P., Mijares, E., Savio, G., Borrego, M., Martínez-Montes, E. Torres, A., 2012. Maturational time course of the envelope following response to amplitude-modulated acoustic signals in rats. Int. J. Audiol. 51, 309-316. https://doi.org/10.3109/14992027.2011.639812. 
Prat, Y., Taub, M., Yovel, Y., 2015. Vocal learning in a social mammal: demonstrated by isolation and playback experiments in bats. Sci. Adv. 1, 1-6. https://doi.org/ 10.1126/sciadv.1500019.

Rance, G., Tomlin, D., Rickards, F.W., 2006. Comparison of auditory steady-state responses and tone-burst auditory brainstem responses in normal babies. Ear Hear. 27, 751-762. https://doi.org/10.1097/01.aud.0000240491.68218.

Razak, K.A., Fuzessery, Z.M., 2007. Development of functional organization of the pallid bat auditory cortex. Hear. Res. 228, 69-81. https://doi.org/10.1016/ j.heares.2007.01.020.

Rübsamen, R., Neuweiler, G., Marimuthu, G., 1989. Ontogenesis of tonotopy in inferior colliculus of a hipposiderid bat reveals postnatal shift in frequencyplace code. J. Comp. Physiol. 165, 755-769. https://doi.org/10.1007/BF00610874.

Ryugo, D.K. Montey, K.L., Wright, A.L., Bennett, M.L, Pongstaporn, T., 2006. Postnatal development of a large auditory nerve terminal: the endbulb of Held in cats. Hear. Res. 216-217, 100-115. https://doi.org/10.1016/j.heares.2006.01.007.

Sanes, D.H., Constantine-Paton, M. 1985. The development of stimulus following in the cochlear nerve and inferior colliculus of the mouse. Dev. Brain Res. 22 255-267. https://doi.org/10.1016/0165-3806(85)90177-4.

Simmons, A.M., Boku, S., Riquimaroux, H., Simmons, J.A., 2015. Auditory brainstem responses of Japanese house bats (Pipistrellus abramus) after exposure to broadband ultrasonic noise. J. Acoust. Soc. Am. 138, 2430-2437. https://doi.org/ 10.1121/1.4931901.

Simmons, A.M., Hom, K.N., Warnecke, M., Simmons, J.A., 2016. Broadband noise exposure does not affect hearing sensitivity in big brown bats (Eptesicus fuscus). J. Exp. Biol. 219, 1031-1040. https://doi.org/10.1242/jeb.135319.

Smith, A.J., Owens, S., Forsythe, I.D., 2000. Characterisation of inhibitory and excitatory postsynaptic currents of the rat medial superior olive. J. Physiol. 529, 681-698. https://doi.org/10.1111/j.1469-7793.2000.00681.x.

Sonntag, M., Englitz, B., Kopp-Scheinpflug, C., Rübsamen, R., 2009. Early postnatal development of spontaneous and acoustically evoked discharge activity of principal cells of the medial nucleus of the trapezoid body: an in vivo study in mice. J. Neurosci. 29, 9510-9520. https://doi.org/10.1523/JNEUROSCI.137709.2009.

Sterbing, S.J., 2002. Postnatal development of vocalizations and hearing in the phyllostomid bat, Carollia perspicillata. J. Mammal. 83, 516-525. https:// doi.org/10.1644/1545-1542(2002)083<0516:pdovah>2.0.co;2.

Tyack, P.L., 2016. Vocal learning and auditory-vocal feedback. In: Vertebrate Sound Production and Acoustic Communication. Springer, pp. 261-295.

Vater, M., Foeller, E., Mora, E.C., Coro, F., Russell, I.J., Kössl, M., 2010. Postnatal maturation of primary auditory cortex in the mustached bat, Pteronotus parnellii. J. Neurophysiol. 103, 2339-2354. https://doi.org/10.1152/jn.00517.2009.

Venkataraman, Y., Bartlett, E.L., 2013. Postnatal development of synaptic properties of the GABAergic projection from the inferior colliculus to the auditory thalamus. J. Neurophysiol. 109, 2866-2882. https://doi.org/10.1152/jn.00021.2013.

Venkataraman, Y., Bartlett, E.L., 2014. Postnatal development of auditory centra evoked responses and thalamic cellular properties. Dev. Neurobiol. 74, 541-555. https://doi.org/10.1002/dneu.22148.

Walker, B.A., Gerhards, C.M., Werner, L.A., Horn, D.L., 2019. Amplitude modulation detection and temporal modulation cutoff frequency in normal hearing infants. J. Acoust. Soc. Am. 145, 3667-3674. https://doi.org/10.1121/1.5111757.

Walsh, E.J., McGee, J., Javel, E., 1986. Development of auditory-evoked potentials in the cat. III. Wave amplitudes. J. Acoust. Soc. Am. 79, 745-754. https://doi.org/ $10.1121 / 1.393463$

Wenstrup, J.J., 1984. Auditory sensitivity in the fish-catching bat, Noctilio leporinus. J. Comp. Physiol. 155, 91-101. https://doi.org/10.1007/BF00610934.

Wu, Shu Hui, Oertel, D., 1987. Maturation of synapses and electrical properties of cells in the cochlear nuclei. Hear. Res. 30, 99-110. https://doi.org/10.1016/03785955(87)90187-0. 